\title{
"Atypical": a typical portrayal of Autism?
}

In conjunction with the rise in prevalence of autism spectrum disorders (ASD) there has been a boom in portrayals of characters in films and TV series who sit somewhere along, or in close proximity to, the autism spectrum. These media portrayals may have negative consequences such as strengthening of stereotypes, stigma and so on. However, such portrayals can also contribute to raising awareness about the condition. Launched in August, the new drama/comedy TV series "Atypical" places Sam Gardner (Keir Gilchrist) centre stage, an 18-year-old high-school student with ASD. At present, 8 episodes have been released and, as is the case for many portrayals of ASD on screen, the accuracy of the presentation is the subject of intense debate. Large numbers of autistic people have been critical of the show and of the choice to cast a non-autistic actor - but is the resulting portrayal inaccurate? In fact, "Atypical” resembles other TV series and films portraying characters on the autism spectrum, in that one gets the feeling of watching a textbook example of the condition. For instance, within the first two minutes of the first episode the main character has neatly described himself in line with the DSM-5 ASD criteria. This is a criticism that can be raised about many such portrayals - research from our group has found autistic characters in TV and film to be archetypal rather than authentic. This medical-textbook-inspired characterisation may explain some of the disapproval from the autistic community. Nevertheless, Sam does have an ASD diagnosis and portraying him as having difficulties in social communication as well as stereotypical behaviour, and focused interests (in this case, in Antarctica) should not come as a surprise for the viewer.

Although "Atypical" is a TV show focused on Sam as the main character, other stories are also being told, offering a glimpse into the everyday challenges and joys that accompany having an autistic teenager in the family. The family situation is perhaps also on the stereotypical side, with a mother completely immersed in her son's autism and a father who has difficulties engaging with his son. That being said, many scenes, especially between Sam and his father (Michael Rapaport), are truly touching and well-played.

Another aspect worth mentioning is savantism. This tends to be a highlighted feature in many autistic character portrayals. Although there are slight hints of savantlike skills in Sam's highly structured drawings in his notebook, this is not overemphasised. 
"Atypical" does well in tackling the myth that people on the spectrum are not interested in dating and sex. Also, some may hopefully change their misconceptions that people with ASD are not interested in social engagement, finding instead that a reluctance to engage has more to do with past experiences and uncertainty in reading social situations.

"Atypical" is well worth a look and offers the promise of becoming a valuable TV show in terms of raising awareness of autism spectrum disorders. But every fictional TV and film portrayal of persons with ASD must come with a warning: as the term "spectrum" emphasises, ASD is a state of neurodiversity that encompasses a vast heterogeneity.

Hence the slightly worn-out saying that 'if you have met one person with autism, you have met one person with autism' equally applies to autistic character portrayals like Sam Gardner in "Atypical”. A TV series like "Atypical” can never be representative for the whole spectrum nor do fully justice to the variety and complexity of autistic lived experiences.

Anders Nordahl-Hansen, PhD (Department of Special Needs Education, University of Oslo)

Nordahl-Hansen, A., Tøndevold, M., \& Fletcher-Watson, S. (2017). Mental health on screen: a DSM-5 dissection of portrayals of Autism Spectrum Disorders in film and TV. Psychiatry Research.

http://dx.doi.org/10.1016/j.psychres.2017.08.050

Conn, R., Bhugra, D., 2012. The portrayal of autism in hollywood films. Int. J. Cult. Ment. Health 1, 54-62.

http://www.tandfonline.com/doi/abs/10.1080/17542863.2011.553369

Bhugra, D. (2003). Teaching psychiatry through cinema. The Psychiatrist, 27(11), 429-430.

http://pb.rcpsych.org/content/27/11/429

Baron-Cohen, S. (2015). Autism, maths, and sex: the special triangle. The Lancet Psychiatry, 2(9), 790-791.

http://www.thelancet.com/journals/lanpsy/article/PIIS2215-0366(15)00397-1/fulltext

Garner, A., Jones, S., \& Harwood, V. (2015). Authentic representations or stereotyped 'outliers': using the CARS2 to assess film portrayals of Autism 
Spectrum Disorders. International Journal of Culture and Mental Health, 8(4), 414-425.

http://www.tandfonline.com/doi/abs/10.1080/17542863.2015.1041993 